\title{
Effects of Indigo Carmine on Human Chondrocytes In Vitro
}

\author{
Timo Zippelius, Arnd Hoburg, Bernd Preininger, Pauline Vörös, Carsten Perka, Georg Matziolis \\ and Eric Röhner ${ }^{*}$
}

Department of Traumatology and Orthopedics, Charité - Universitätsmedizin, Berlin, Germany

\begin{abstract}
Joint infections following or accompanying superficious soft tissue infections are severe complication in orthopedic surgery. The use of intra-articular blue staining is a helpful method to visualize a fistula and to differentiate between superficial and intra-articular infections. Regarding this clinical implication data about the effects of indigo carmine, a frequently used blue staining substance, on cartilage is missing. The hypothesis of this study was that indigo carmine damages human chondrocytes in a time and concentration dependent manner. Human chondrocytes were isolated from donors with osteoarthritis who were treated with TKA. Cells were cultivated and treated with different concentrations of indigo carmine for 5 and 10 minutes. Morphologic damage was examined by light microscopy. Toxicity was quantified by counting vital cell number and lactate dehydrogenase (LDH) expression. Analysis by light microscopy showed defected cell structure and loss of cell number after treatment with $100 \%$ indigo carmine for 10 minutes. Treatment with $10 \%$ and $1 \%$ indigo carmine showed no significant cell defects and loss of cells. Counting vital cell number showed loss of vital cells after treatment with $100 \%$ and $10 \%$ indigo carmine for 10 minutes. LDH expression was significantly increased after treatment with $100 \%$ indigo carmine.

Toxic effects were shown after treatment with indigo carmine. Therefore, it should be used in 1:100 dilution. This is both, sufficient for visualizing a fistula in a possible clinical application and could be protective for chondrocytes.
\end{abstract}

Keywords: Indigo carmine, human chondrocytes, toxicity.

\section{INTRODUCTION}

The use of blue staining with indigo carmine (IC) is a helpful technique in orthopedic and trauma surgery. It is used for coloring cystic lesions of knee or hip, visualization of ganglia before resection or staining of degenerative nucleus pulposus before endoscopic intervertebral nucleotomy [1-3]. Another field of application is the blue dyeing of traumatic opened bursa for coloring and saving the right area of operation. After staining the bursa can be removed completely.

We frequently apply IC to differ between superficial and intra-articular infections. Based on the result the superficial infection is either treated without opening of the joint or the underlying joint infection must be addressed by arthroscopic lavage and additional synovectomy depending on the stage of the infection [4].

It is known that intra-articular joint injections with local anesthetics combined with or without corticosteroids can induce chondrolysis. Various studies have demonstrated toxic effects of commonly used local anesthetics and steroids like bupivacaine, lidocaine, ropivacaine or triamcinolone on articular cartilge in vivo and in vitro [5-8].

To the present time effects of IC on human cells especially human chondrocytes are rarely examined and documented in current literature.

*Address correspondence to this author at the Center for Musculoskeletal Surgery, Orthopedic Department, Charité University Hospital, Berlin, Charitéplatz 1, D-10117 Berlin, Germany; Tel: ++49 (0)30 450652 463; Fax: ++49(0)30 450515 911; E-mail: eric.roehner@charite.de
The aim of this study is to show that IC has toxic effects on human chondrocytes in vitro. We hypothesize that different concentrations of IC can significantly induce cell death after short incubation times of 5 and 10 minutes.

\section{MATERIALS AND METHODOLOGY}

Tissue culture plasticware was obtained from TPP (Trasadingen, Switzerland). Culture medium, phosphate buffer saline (PBS), fetal calf serum (FCS), trypsin, penicilline and streptomycine were purchased from Biochrom (Berlin, Germany). All other reagents were obtained from Sigma-Aldrich (Deisenhofen, Germany).

\section{Chondrocyte Isolation, Culture and Treatment}

Chondrocyte isolation was performed as described before $[9,10]$. Cartilage was obtained from 6 human donors ( 4 men and 2 women) with knee osteoarthritis not presenting any kind of infectious signals (normal $\mathrm{CrP}<5 \mathrm{mg} / 1$, leukocytes $<$ $12000 / \mu 1$, normal temperature $<37,5^{\circ} \mathrm{C}$ ). Experimental protocols were approved by the local ethics committee. Cartilage was rinsed and crushed and then minced and digested in medium containing $1 \mathrm{mg} / \mathrm{ml}$ pronase (SigmaAldrich, Deisenhofen, Germany) for 30 minutes at $37^{\circ} \mathrm{C}$. After then, digestion medium was discarded and the tissue was digested with medium containing $1 \mathrm{mg} / \mathrm{ml}$ clostridial collagenase (Sigma-Aldrich, Deisenhofen, Germany) at $37^{\circ} \mathrm{C}$ over night. Digested solution was filtered $(70 \mu \mathrm{m}$ Nylon, BD Falcon, Bedford, Germany) and centrifuged at $1200 \mathrm{rpm}$ for eight minutes. The supernatant was discarded and the cell pellet was washed 3 times with phosphate buffer saline (PBS). Then chondrocytes were suspended in DMEM Hams-F12 with $10 \%$ FCS, $1 \%$ penicillin/ streptomycine and 
cultured at $37^{\circ} \mathrm{C}, 95 \%$ air and $5 \% \mathrm{CO}^{2}$. Human chondrocytes were cultured and grown on 24-well plates at a density of sub-confluence, were added with $200 \mu 1$ of $1 \%, 10 \%$ and $100 \%$ solutions of $0.8 \%$ indigo carmine solution (Akorn Inc., Lake Forest, IL, USA) for 5 and 10 minutes. PBS treated chondrocytes were used as negative control. Chondrocytes treated with $2 \%$ Triton X 100 were used as positive damaged control chontrocytes (as described before $[9,10])$. The experiments were performed immediately.

\section{Detection of Cell Structure}

Human chondrocytes grown on 6-well plates to $80 \%$ subconfluence were incubated with different concentrations of IC for 5 and 10 minutes. After treatment the results were interpreted with light microscopy analysis (Axiovert $40 \mathrm{C}$ Light Microscope, lens 10x0.25, ocular 10x18 Zeiss, Göttingen, Germany). The view fields were then digitized by a digital camera (Canon EOS 500D, 15.1 Megapixels).

\section{Determination of Vital Cell Number}

Human chondrocytes grown on 24-well plates at a density of $2 \times 10^{4}$ cells per well, were incubated with different concentrations $(1 \%, 10 \%, 100 \%)$ of IC, PBS (negative control) and Triton X 100 (positive control) for 5 and 10 minutes and were counted by using Casy Cell-Counter. After removing of solutions of IC, all cells were detached with 100 $\mu 1$ trypsin. The detection of living cell numbers was determined by using Casy Cell-Counter and Analyser System (Schärfe-System, Reutlingen, Germany).

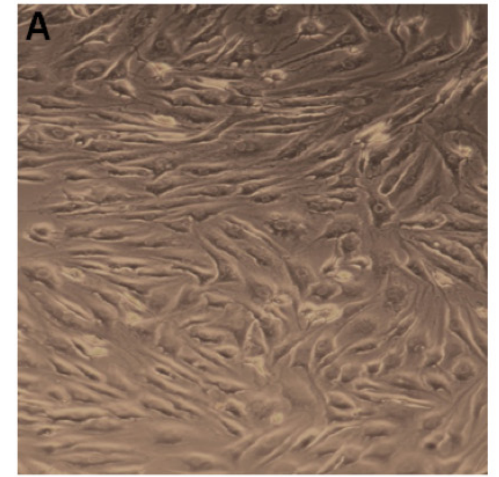

\section{Activity of Lactate Dehydrogenase (LDH)}

LDH activity is a marker of advanced cell death. Release of LDH exposure indicates the loss of membrane plasma integrity as possible marker of increased cell necrosis at this stage. Chondrocytes monolayer were treated with different concentrations of IC solutions, PBS (negative control) and Triton X 100 (used as positive control) for 5 and 10 minutes. LDH activity in the supernatant was determined by the colorimetric measurement of the reduction of sodium pyruvate in the presence of nicotinamide adenine dinucleotide hydrogene (NADH) and expressed as the percentage of total enzyme activity liberated from chondrocytes in the presence of the antiseptics.

\section{Statistical Analysis}

A nonparametric Wilcoxon matched-pairs test was used as indicated in the legends. A $p$ value of $<0.05$ was considered to be significant.

\section{RESULTS}

Human chondrocytes treated with solutions of $100 \%$ IC showed increased number of cells with defect cell structure (Fig. 1E). Chondrocytes showed loss of cell contacts after an incubation time of 10 minutes as revealed by light microscopy. There was no defective cell structure of chondrocytes when cultured with PBS (control) (Fig. 1A). The incubation with $2 \%$ Triton X100 as known mediator of necrosis revealed clear cell damage and induction of necrosis in human chondrocytes (Fig. 1B). The treatment with 10 and

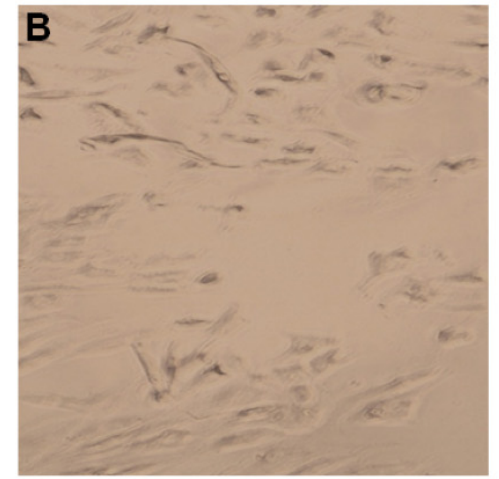

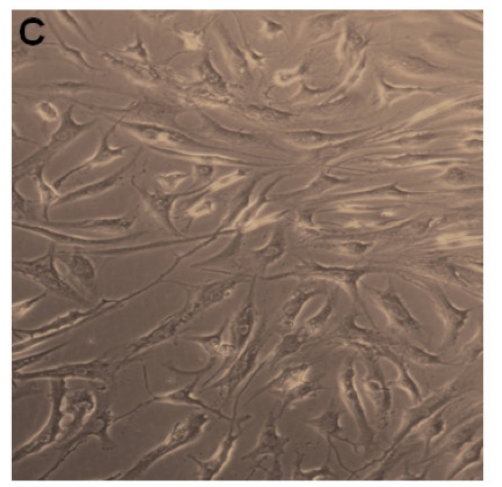
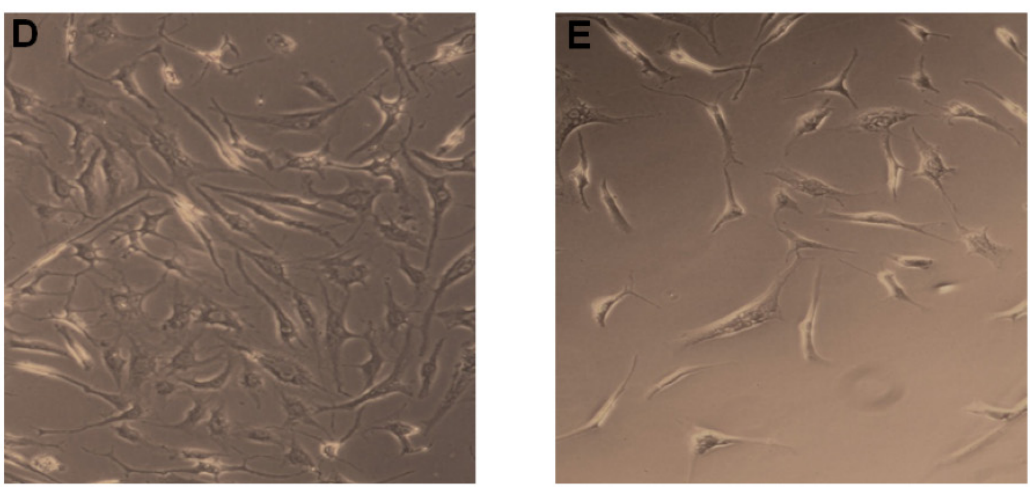

Fig. (1). Chondrocyte damage after exposure to IC. Chondrocytes were treated with IC and were analyzed by light microscopy. (A) Untreated control chondrocytes. (B) Chondrocytes treated with Triton X100, a known inducer of cell necrosis. (C) Chondrocytes treated with 1\% IC. (D) Chondrocytes incubated with 10\% IC. (E) Chondrocytes treated with $100 \%$ IC. One representative result is shown from at least three independently performed experiments. 
$1 \%$ IC showed mostly intact cell structure with not significantly loss of cell number (Fig. 1C, D).

The determination of vital cell numbers of $100 \%$ IC treated chondrocytes showed decrease of vital cells after a short incubation time of 5 minutes (100\% IC vs ctr: $16000 \pm 1000 v s$ $19000 \pm 1000, \mathrm{p}<0.05)$ and 10 minutes $(100 \%$ IC $v s$ ctr: $150000 \pm 3000$ vs $19000 \pm 1000, \mathrm{p}<0.05)$. Concentrations of $10 \%$ IC reduced cell number significantly after 10 minutes $(10 \%$ IC vs ctr: $15000 \pm 3000$ vs $19000 \pm 1000, \mathrm{p}<0.05$, Fig. $(\mathbf{2 A}, \mathbf{B})$. The treatment with $1 \%$ IC showed no significant reduction of vital cell number (Fig. 2A, B).

LDH activity was analyzed in the supernatant of each cell culture. Compared to control, significant LDH release was noticed already after 5 and 10 minutes of treatment with $100 \%$ IC indicating beginning necrotic cell death at that stage (10minutes: $100 \%$ IC vs ctr: $67 \% \pm 4 \%$ vs $55 \% \pm 6 \%$ and 5 minutes: $62 \% \pm 4 \%$ vs $55 \% \pm 6 \%, \mathrm{p}<0.05)$. We detected no LDH release at each time after incubation with 10 or $1 \%$ IC after 5 or 10 minutes (Fig. 3A, B).

\section{DISCUSSION}

The present study shows toxic effects of 100 and $10 \%$ indigo carmine on human chondrocytes. IC increased LDH expression and reduced vital cell number after a short treatment time of 10 minutes.

IC is traditionally used as $\mathrm{pH}$ indicator or food colorant. In medicine IC is applied as marker dye for cystoscopy, to detect amniotic fluid leaks, for differential diagnosis between non-neoplastic and neoplastic colorectal lesions, dying sentinel lymph node or intra-operative tissue staining of invaded oral carcinoma [11-16].

In orthopedic and trauma surgery, the use of IC is limited but has presently gained increasing interests. Despite the large application of IC in general surgery, toxic effect on human tissue is rarely documented in literature.

The study group of Gül et al. could show that methylene blue (MB) and IC induce adhesions in the abdominal or pelvic cavity in comparison to autologous erythrocyte suspension. Thirty healthy female nulliparous Wistar Albino rats were included and received a $1 \mathrm{ml}$ i.p. injection of 5\% methylene blue solution, $5 \%$ IC solution or $5 \%$ fresh autologous erythrocyte suspension. Next to the induction of intra-abdominal and peritoneal adhesions by $\mathrm{MB}$ and IC, an increased activation of macrophages could be shown by MB and IC [17].

In a work of Sheynkin et al. the effects of commonly used intra-operative vasography and tissue staining agents, indigo carmine, methylene blue, and Renografin on sperm motility were evaluated. In this study Semen from 20 healthy men were mixed with undiluted and diluted of standard solutions of indigo carmine, methylene blue, and Renografin. Then sperm motility was assessed. A significant decrease of sperm motility could be demonstrated by undiluted and $2 \mathrm{x}$ diluted indigo carmine [15].

In the present work direct effects of IC on human chondrocytes were examined. We were able to show a reduction of cell number and defective cell structure after treatment with $100 \%$ IC more than $10 \%$ or $1 \%$ IC. This could result from cell damages seen by beginning of cell necrosis [18, 19].

Detection of LDH activity showed a significant increase of LDH activity already after a short incubation time of 5 and 10 minutes with $100 \%$ IC. No LDH activity was detected after treatment with 10 and $1 \%$ IC. Increase of LDH activity after the treatment with $100 \%$ IC indicated the loss of membrane plasma integritiy as possible beginning of cell death $[18,19]$.

In in vitro studies with human cells uncontrollable influences may play an important role such as deviation temperature, barometric pressure or contamination. Therefore, this cell model may have limitations. The use of human chondrocytes from cartilage of patients with knee osteoarthritis yields not fully intact and healthy cartilage tissue, which one would find in healthy people without osteoarthritis. The results of our study do not represent in vivo situation, as in vitro results show higher cell toxicity than in vivo examinations based on the direct chondrocyte treatment without contact to blood. Additionally IC solutions in vitro do not have to pass different barriers like chondrocytes matrix.

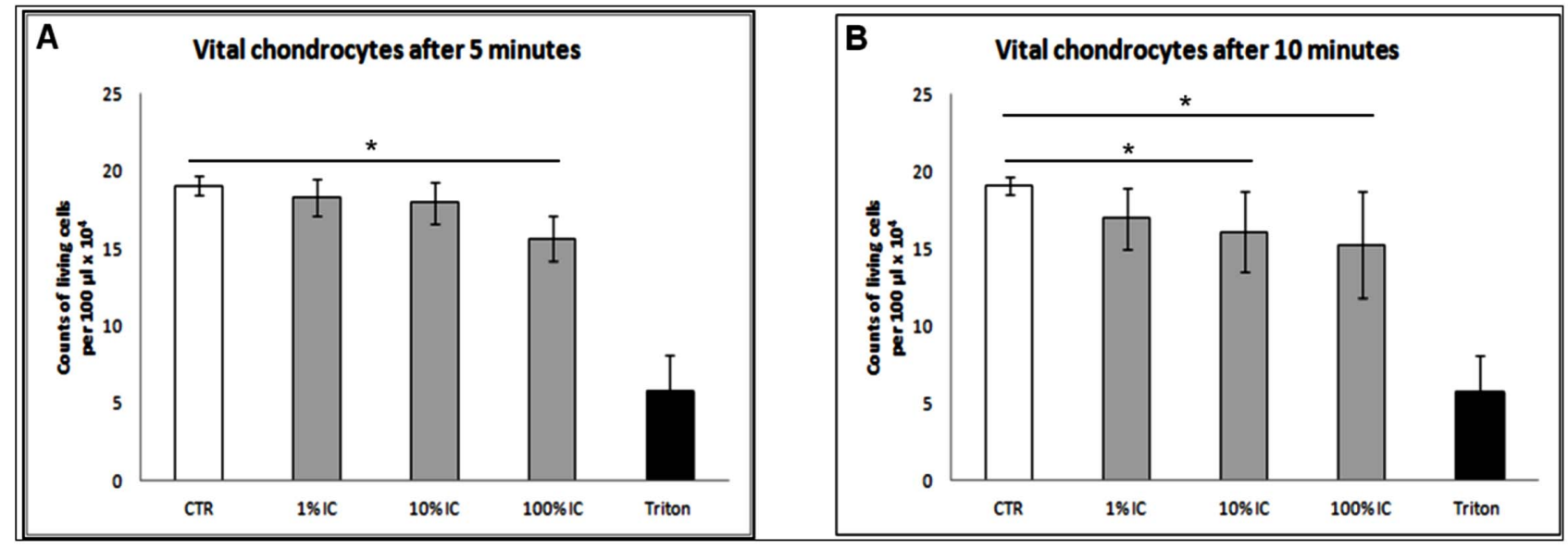

Fig. (2). Determination of vital cells after 5 and 10 minutes versus negative control. (A) Significant decrease of vital chondrocytes after treatment with $100 \%$ IC for 5 minutes. (B) Significant decrease of living cells after treatment with $10 \%$ and $100 \%$ IC for 10 minutes. (A, B) $\mathrm{n}=6$, Mean \pm SEM, A nonparametric Wilcoxon matched pairs test was used as indicated in the legends. A p value of $<0.05$ was considered to be significant. 


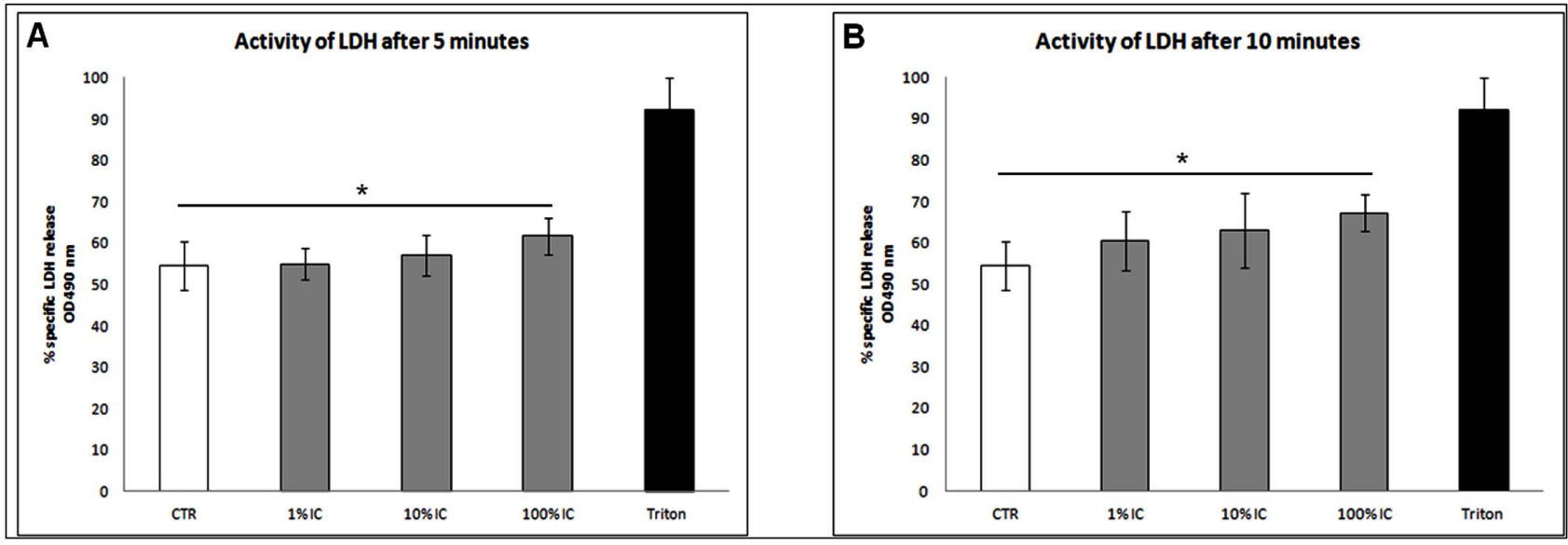

Fig. (3). LDH release following treatment with IC. After the indicated time points supernatants were analyzed for LDH by ELISA. (A, B) Significant increase of LDH activity after treatment with $100 \%$ IC for 5 and 10 minutes. $n=6$, Mean \pm SEM, A nonparametric Wilcoxon matched pairs test was used as indicated in the legends. A p value of $<0.05$ was considered to be significant.

\section{CONCLUSION}

In summary toxic effects of 100 and $10 \%$ IC on human chondrocytes could be shown after short incubation times of five and 10 minutes. In consideration of our results and the results of studies found in literature toxic effects of IC are rarely documented and need further investigations. Therefore IC should only be applied in a dilution of 1:100 for a short time. To the present long time effects of IC are not known. To prevent possible cartilage damage an additional rinsing of IC is recommend.

\section{CONFLICT OF INTEREST}

The authors confirm that this article content has no conflict of interest.

\section{ACKNOWLEDGEMENTS}

Declared none.

\section{REFERENCES}

[1] Kim IS, Kim KH, Shin SW, Kim TK, Kim JI. Indigo carmine for the selective endoscopic intervertebral nucleotomy. J Korean Med Sci 2005; 20: 702-3.

[2] Park HJ, Lee SM, Choi JA, Park NH, Kim HS, Park SI. Preoperative localization of cystic lesions in the knee using ultrasound-guided injection of indigo carmine. J Clin Ultrasound 2010; 38: 305-8.

[3] Yao J, Trindade MCD. Color-aided visualization of dorsal wirst ganglion stalks aids in complete arthroscopic excision. Arthroscopy 2011; 27: 425-29.

[4] Stutz G, Gächter A. Diagnostik und stadiengerechte Therapie von Gelenkinfekten. Unfallchirurg 2001; 104: 682-86.

[5] Syed HM, Green L, Bianski B, Jobe CM, Wongworawat MD. Bupivacaine and triamcinolone may be toxic to human chondrocytes. Clin Orthop Relat Res 2001; 469: 2941-2947.
[6] Chu CR, Coyle $\mathrm{CH}$, Chu CT, et al. In vivo effects of single intraarticular injection of $0.5 \%$ bupivacaine on articular cartilage. J Bone Joint Surg Am 2010; 92: 599-608.

[7] Lo IKY, Sciore P, Chung M, et al. Local anesthetics induce chondrocyte death in bovine articular cartilage disks in a dose-and duration-dependent manner. Arthroscopy 2009; 25: 707-15.

[8] Piper SL, Kramer JD, Kim HT, Feeley BT. Effects of local anesthetics on articular cartilage. Am J Sports Med 2011; 39: 2245-53.

[9] Röhner E, Seeger JB, Hoff P, et al. Preferred use of polyhexanide in orthopedic surgery. Orthopedics 2011; 34: 664-8.

[10] Röhner E, Kolar P, Seeger JB, et al. Toxicity of antiseptics against chondrocytes: what is best for the cartilage in septic joint surgery? Int Orthop 2011; 35: 1719-23.

[11] Fu KI, Sano Y, Kato S, et al. Chromoendoscopy using indigo carmine dye spraying with magnifying observation is the most reliable method for differential diagnosis between non-neoplastic and neoplastic colorectal lesions: a prospective study. Endoscopy 2004; 36: 1089-93.

[12] Nagao T, Kinoshita T, Hojo T, Kurihara H, Tsuda H. Sentinel lymph node biopsy using indigo carmine blue dye and the validity of ' $10 \%$ rule and 4 nodes rule. Breast 2012; 21(4): 455-8.

[13] Kosaka T, Asano T, Azuma R, Yoshii H, Yamanaka Y, Hayakawa M. A case of vesicocutaneous fistula to the thigh. Urology 2009; 73: 929.e7-8.

[14] Kurita H, Kamata T, Koike T, Kobayashi H, Kurashina K Intraoperative tissue staining of invaded oral carcinoma. Pathol Oncol Res 2008; 14: 461-5.

[15] Sheynkin YR, Starr C, Li PS, Goldstein M. Effect of methylene blue, indigo carmine, and Renografin on human sperm motility. Urology 1999; 53: 214-7.

[16] Mahadevan MM, Weitzman GA, Hogan S, Breckinridge S, Miller MM. Methylene blue but not indigo carmine is toxic to human luteal cells in vitro. Reprod Toxicol 1993; 7: 631-3.

[17] Gül A, Kotan C, Dilek I, Gül T, Tas A, Berktas M. Effects of methylene blue, indigo carmine solution and autologous erythrocyte suspension on formation of adhesions after injection into rats. J Reprod Fertil 2000; 120: 225-9.

[18] Bühling K, Lepenies J, Witt K. Intensivkurs Allgemeine und spezielle Pathologie. Auflage: Urban und Fischer, München 2004; vol. 3.

[19] Walker NI, Harmon BV, Gobe GC, Kerr JV. Patterns of cell death. Methods Achiev Exp Pathol 1988; 13: 18-54. 